\title{
Covert inhalation of tablet causing fatal pneumonia
}

\author{
S.N. Hill, A.N.H. Main and W.J. Fuggle \\ Department of Geriatric Medicine, University of Birmingham, and Department of Pathology, Selly Oak \\ Hospital, Raddlebarn Road, Birmingham B29 6JD, UK.
}

\begin{abstract}
Summary: Inhalation of foreign bodies is usually associated with acute respiratory symptoms. We report a case of an elderly woman who died from pneumonia due to covert tablet inhalation. Clinicians caring for the elderly should consider this as a possible cause for pneumonia which fails to respond to usual therapy.
\end{abstract}

\section{Introduction}

The incidence of foreign body inhalation into the tracheobronchial tree in hospital chest units is about twenty cases annually, the majority of patients being children. ${ }^{1,2}$ There is usually a history of inhalation or acute respiratory symptoms. ${ }^{3,4} \mathrm{We}$ report an elderly woman with unsuspected tablet inhalation causing fatal pneumonia.

\section{Case report}

A 79 year old woman entered hospital after six weeks' anorexia, night sweats, general malaise and exertional breathlessness. She had received a 10 day course of oral ampicillin, and a further course of oral flucloxacillin and ampicillin (Magnapen) which continued until her admission. Her other medication had been methyl dopa $250 \mathrm{mg}$ four times a day, hydrochlorothiazide and amiloride (Moduretic) daily, ferrous sulphate (Ferrogradumet) and diphenoxylate hydrochloride (Lomotil) as required.

Examination revealed an ill-looking woman, breathless on slight exertion. At the base of the right lung there were coarse inspiratory crepitations and moderate dullness to percussion.

Investigations revealed haemoglobin $8 \mathrm{~g} / \mathrm{dl}$, erythrocyte sedimentation rate $126 \mathrm{~mm} /$ hour, neutrophil leucocytosis $\left(17.2 \times 10^{9} / 1\right)$ and mild renal failure. Serum electrophoresis showed no evidence of myelomatosis. Blood culture showed no growth. Sputum specimens were mucoid and blood stained with no bacterial growth or malignant cells. Chest $\mathrm{X}$-ray showed consolidation of the posterior basal segment of the right lower lobe.

Correspondence: S.N. Hill, M.B., M.R.C.P.

Accepted: 25 June 1987
She remained unwell with fever and night sweats despite treatment with oral erythromycin and metronidazole. Obstructing bronchial carcinoma causing distal lobular pneumonia was suspected. We therefore felt justified in performing bronchoscopy to achieve a definite diagnosis but before this could be performed the patient suffered a stroke complicating her worsening pneumonia and died 2 days later, four weeks after admission.

Autopsy revealed massive consolidation of the right lower lobe. The right lower lobe bronchus was completely occluded by impacted hard material, the appearance being that of a tablet. Histology of the lungs confirmed pneumonia and showed no evidence of neoplasm or arteritis.

\section{Discussion}

Old people are major tablet consumers and are most susceptible to illness which impairs swallowing or ability to report tablet inhalation. This patient was mentally clear with normal swallowing. The nature of an inhaled object reflects the patients' culture and eating habits. ${ }^{3}$ Great numbers of tablets are swallowed in this country yet inhalation has not been reported often. In one unusual case ${ }^{5}$ an asthmatic adult inhaled a wrapped Lynestrenol tablet inadvertently from his Medihaler, but gave a clear history of the tablet entering his lung. Unsuspected inhalation of other objects is well recognized $^{6,7}$ and, particularly when vegetable in origin, may result in lung sepsis. ${ }^{3}$ Most foreign bodies are found in the right lung, particularly in adults. $^{2}$

Removal of foreign bodies by rigid bronchoscopy under general anaesthetic with adequate preoxygenation, followed by physiotherapy and

(C) The Fellowship of Postgraduate Medicine, 1988 
antibiotics, has been shown to be safe and effective, usually obviating the need for surgery. ${ }^{1,3}$ Early bronchoscopy in this case might have allowed removal of the tablet and effective treatment of the pneumonia. We would remind clinicians caring for old people to be aware of the possibility of covert

\section{References}

1. Osama, A. Aspirated foreign bodies in the tracheobronchial tree: Report of 250 cases. Thorax 1976, 31: 635-640.

2. Danuludis, J., Symeonidis, B., Triaridis, K. \& Kouloulas, A. Foreign body in the airway - a review of 90 cases. Arch Otolaryngol 1977, 103: 570-573.

3. Editorial. Inhaled foreign bodies. $\mathrm{Br} \mathrm{Med} J$ 1981, 282: 1649-1650.

4. Griffiths, D.M. \& Freeman, N.V. Expiratory chest X- tablet inhalation as a rare, but potentially treatable, cause for pneumonia which fails to respond to usual therapy, particularly in the right lower lobe, and to arrange for prompt bronchoscopy where bronchial obstruction is suspected.

ray examination in the diagnosis of inhaled foreign bodies. $\mathrm{Br}$ Med J 1984, 288: 1074-1075.

5. Wiesel, J.M., Bar, Z.G. \& Reinmesser, R. Intrabronchial tablets - Jet propelled. A case report. $J$ Laryngol Otol 1981, 95: 219-220.

6. Ahmad, S. Covert bronchial foreign body. $\mathrm{Br}$ Med J 1984, 289: 1743.

7. Case Records of the Massachusetts General Hospital. N Engl J Med 1983, 309: 1374-1381. 